\title{
Valorization of by-products of fish by reactive extrusion to produce biofuel
}

\author{
Dounia MAKOURE ${ }^{1,2}$, Abdellah ARHALIASS ${ }^{1}$, Adil ECHCHELH ${ }^{2}$, Jack LEGRAND ${ }^{1}$ \\ $\left\{\right.$ dounia.makoure@etu.univ-nantes.fr ${ }^{1,2}$, Abdellah.Arhaliass@ univ-nantes.fr ${ }^{1}$, \\ adilechel@gmail.com ${ }^{2}$ \} \\ ${ }^{1}$ University of Nantes, Laboratoire GEPEA, UMR-CNRS 6144, CRTT, Boulevard de \\ l'Université, 44600, Saint Nazaire, France \\ ${ }^{2}$ University Ibn Tofail, b.p 133 av. université, 14000, Kenitra, Maroc
}

\begin{abstract}
In this paper we present the reactive extrusion process to extract the lipids from by-products of fish. The study focuses on the technical feasibility of using these purified lipids as an additive (between 5 and $40 \%$ dilution) in a diesel fuel for use in an engine.The parameters of extrusion process were optimised in order to extract the most percentage of oil and the results of lipids extraction were compared with other techniques like batch process. The physico-chemical characteristics (viscosity, density, calorific value, acidity index) of extracted and transesterified oil are compared with diesel characteristics. Further tests will concern the production of mixtures of the obtained oil and the diesel oil at different dilutions.
\end{abstract}

Keywords:Reactive extrusion,valorization, by-products, transesterification, biodiesel 


\section{Introduction}

The depletion of oil resources of the planet and the enhancement of laws and regulations promoting "green" fuels oriented the scientific research toward the discovery of new sources of energy. The activities of smoking fish and canning industry generate every year several tons of by-products representing the residue of the fish and crustaceans manufacturing. The valorization of these by-products (residue biomass) constitutes an important issue to allow a more profitable and more sustainable exploitation of the marine resource. It is therefore important to develop new, high value-added ways of promoting these co-products. The main process used is the reactive extrusion process to extract the lipids used as a biofuel. Moreover, the residue marine biomass represents an alternative source for biofuel production. Biofuel represents an alternative to diesel derived from fossil energy. It is a clean energy source that emits less greenhouse gases.,Its combustion is also less dangerous and non-toxic. Biofuel can be used in pure form B100, B20 is a mixture of $80 \%$ diesel and $20 \%$ biodiesel, B5 contains 95\% diesel and 5\% biodiesel [1]. Biodiesel is characterized by physico-chemical properties, the most interesting are viscosity, calorific value, flash point, chemical composition, acidity. The most common method for producing biodiesel is the transesterification of vegetable oils and animal fats[2]-[5].

\section{Materials and methods}

\subsection{Raw material}

The fish co-products used in this study were collected in fish shops. The origin of these coproducts are two types of fatty and medium-fat fish such as salmon, trout and sardine. The enzymes used for hydrolysis are Neutrase and Protamex. they are industrial enzymes produced by genetic engineering by Novozyme AS. The optimal conditions for Protamex are: $\mathrm{pH}$ between 5.5 and 7.5 and temperature between 35 and $60^{\circ} \mathrm{C}$. Protamex can be inactivated by heating to $85^{\circ} \mathrm{C}$ for $10 \mathrm{~min}$. For Neutrasse, its optimal $\mathrm{pH}$ is between 5.5 and 7.5 and its optimal temperature is between 35 and $50^{\circ} \mathrm{C}$. The Neutrasse inactivation is done by heating to $90^{\circ} \mathrm{C}$ for $5 \mathrm{~min}$.

\subsection{Batch enzymatic hydrolysis}

The first enzymatic hydrolysis tests were carried out in a double jacketed reactor to optimize the conditions of the enzymatic hydrolysis reaction (reaction temperature, enzyme type, enzyme/substrate ratio and reaction time).The reactor is composed of a thermal regulation system, $\mathrm{pH}$ and temperature probe to control the reaction parameters and a pneumatic stirrer to homogenize the mixture see Figure1. 


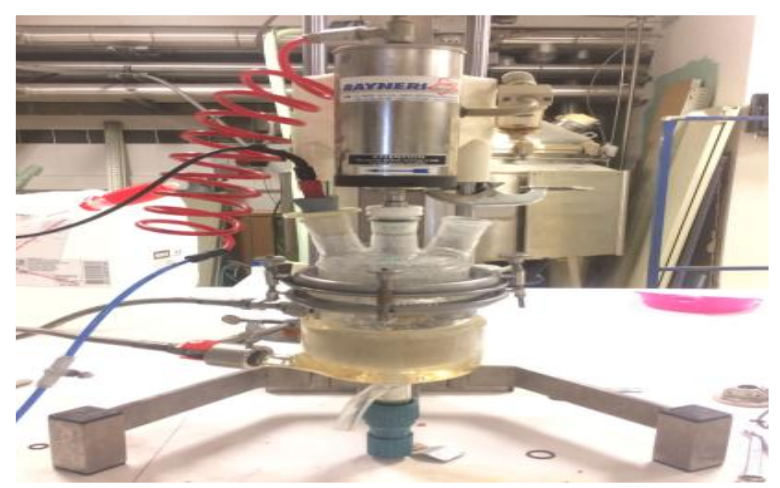

Fig. 1. Batch test equipment

\subsection{Enzymatic hydrolysis by reactive extrusion}

The reactive extrusion is a process that combines the strength of the extruder's mixture with the chemical industry. The extrusion consists in forcing a product to flow into a screw/barrel system called an extruder. The material moves under the action of high pressures of one or more screws that rotate in a barrel. These screws will also compress and shear the material. All the tests were carried out by using corotative twin screw extruder type Clextral $\mathrm{BC} 21$ see figure2.

The extruder is composed of the following components:

-A solid feed hopper equipped with a dosing pump,

-two parallel, co-rotating and interpenetrating screws,

-a thermally regulated barrel and a water circulation cooling system,

-a variable speed motor.

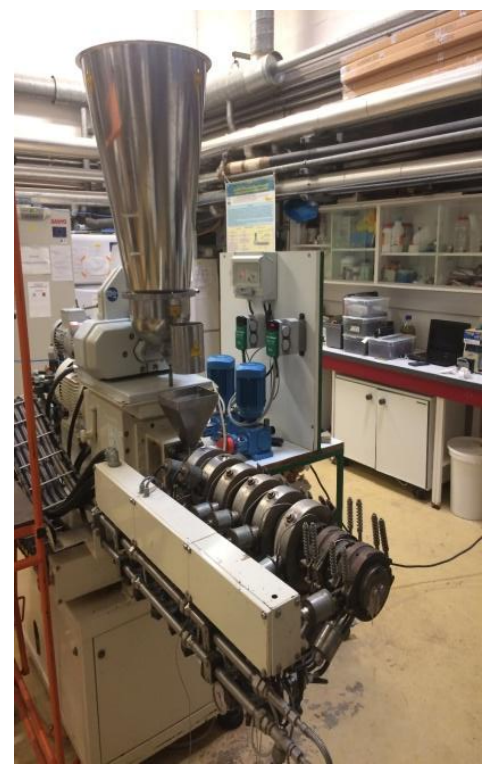

Fig. 2. twin screw extruder type Clextral BC 21 


\subsection{Centrifugation}

Centrifugation is a process of separating mixture compounds due to the difference in densities of each compound by centrifugal force. This step is performed after the enzyme inactivation by using an Awel centrifuge - model CF 20/20-R. The hydrolysate is centrifuged at $4400 \mathrm{rpm}$ (3500g) for 30 minutes at $20^{\circ} \mathrm{C}$.

After centrifugation, four phases are obtained in the tubes (figure 3) :

- The oily phase, containing mainly lipids

- The aqueous phase, containing mainly water

- The oil emulsion phase

- The pellet phase, with insoluble fractions

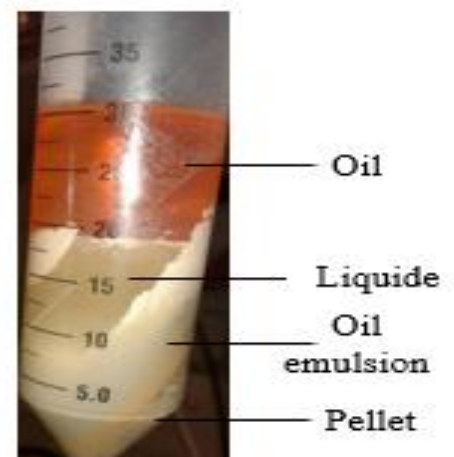

Fig. 3. Phase separation by centrifugation

\subsection{Transesterification reaction}

The fish oil preheated at $40^{\circ} \mathrm{C}$ was added with alkoxide mixture (methanol and sodium hydroxide). The methanol/oil ratio is $6: 1$ and the catalyst amount is about $1 \%$ of the total mass of oil. The alkoxide and oil mixture is then stirred throughout the reaction by a mechanical stirrer for $45 \mathrm{~min}$. At the end of the reaction, the contents are poured into a separatory funnel and left to rest for a few hours.

\section{Results and Discussion}

\subsection{Enzymatic hydrolysis}




\subsubsection{Enzymatic hydrolysis of salmon byproducts}

The first test performed was an enzymatic hydrolysis of salmon co-products in double walled glass reactor to determine the optimal reaction conditions and to compare the two processes. Then the extrusion (without enzyme) and reactive extrusion (with enzyme) processes were applied to show the effect of enzyme in the lipid extraction (figure 4).

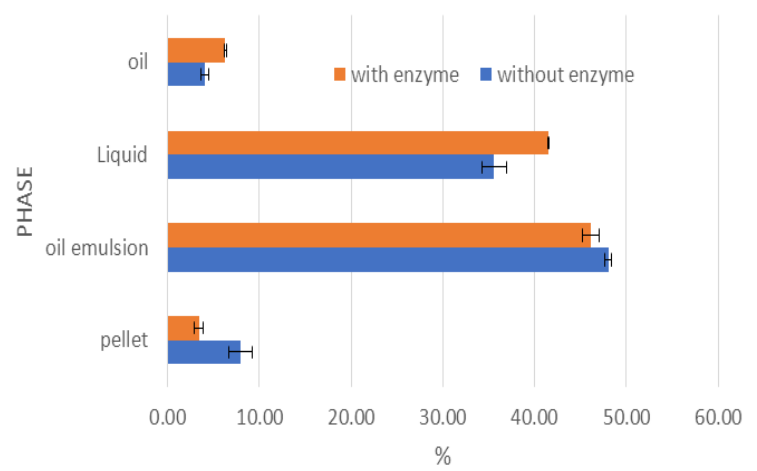

Fig. 4. mass balances of the phases in the extruder with and without enzyme (salmon)

Due to the strength of extruder mixing and the effect of the enzyme in the same time, we got $2.2 \%$ increase of oil recovery than using just the extruder without the presence of an enzyme. The pourcentage of the pellet phase is also reduced by $4.5 \%$ by using the reactive extrusion process. This reduction showed the hydrolysis effect of the enzyme on the raw material.

Batch tests were carried out before the reactive extrusion process. Due to high mixture capacity, the enzymatic hydrolysis in the extruder showed an increase of $3.76 \%$ of oil phase and $12.07 \%$ of oil emulsion phase against to batch process see figure 5 .

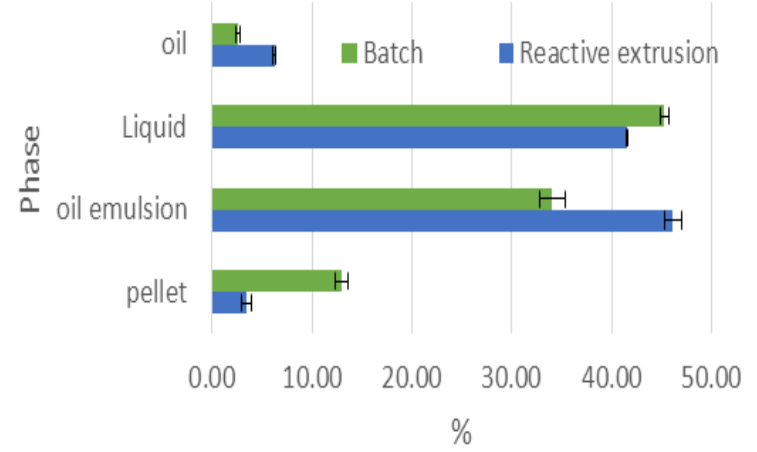

Fig. 5. mass balances of the phases in batch and reactive extrusion (salmon)

\subsubsection{Enzymatic hydrolysis of trout byproducts}


The percentage of the pellet phase is reduced up to $45 \%$ and the oil emulsion phase is increase up to $49.16 \%$ with enzyme consumption in the enzymatic hydrolysis in the extruder see figure 6 . The tests carried out beforehand in the batch process showed a reduction of $20.21 \%$ for the oil emulsion and a small wage decrease of $1 \%$ of oil in comparison with the reactive extrusion see figure 7 . The combination between enzyme and extrusion is more efficient than the batch process and the extrsuion without enzyme because it provides an important denaturation of the byproducts.

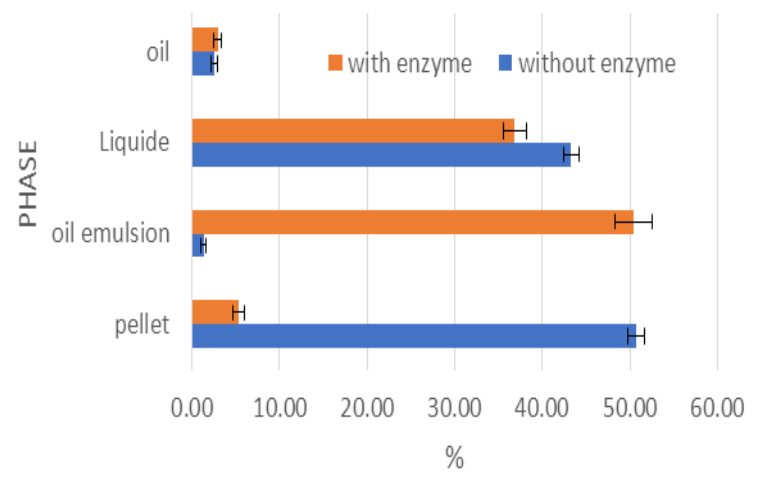

Fig. 6. mass balances of the phases in the extruder with and without enzyme (Trout)

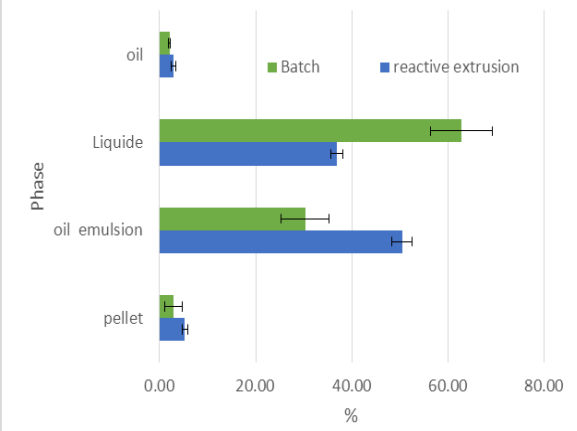

Fig. 7. mass balances of the phases in batch and reactive extrusion (Trout) 


\subsubsection{Enzymatic hydrolysis of sardine byproducts}

There are significant difference between enzymatic hydrolysis in batch and reactive extrusion on oil recovery up to $5 \%$. The percent difference of the oil emulsion phase between the reactive extrusion and the batch is up to $24.05 \%$ (figure 8 ). The difference between the batch and reactive extrusion processes is the extruder screws shear force which improves the contact between the raw material and the enzyme.

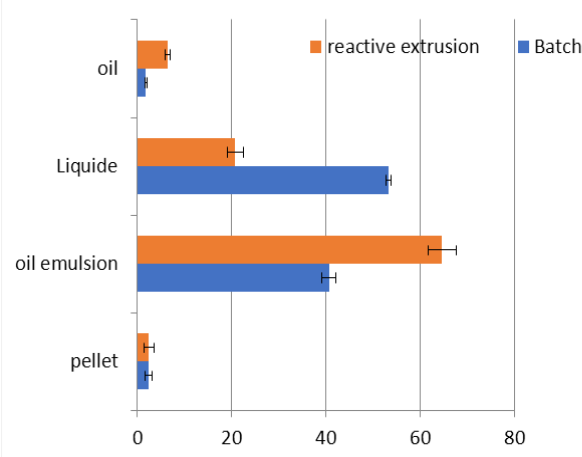

Fig. 8. mass balances of the phases in batch and reactive extrusion (Sardine)

\subsection{Determination of total fatty acids}

The dosages of total fatty acids were carried out by gas chromatograph (GC) of salmon, trout and sardine oil. These analyses allowed to determine the percentage of total fatty acids and their different classifications.

\subsubsection{Different classifications of fatty acids in salmon oil}

From the chromatogram of the salmon oil phase, we notice the existence of 11 peaks including 3 with a major representation for oleic acid (51\%), linoleic acid $(19.92 \%)$ and $\alpha$ linolénique acid (10.03\%) see figure 9 and 10. The salmon oil contains a low level of the erucic acid $(2.2 \%)$.

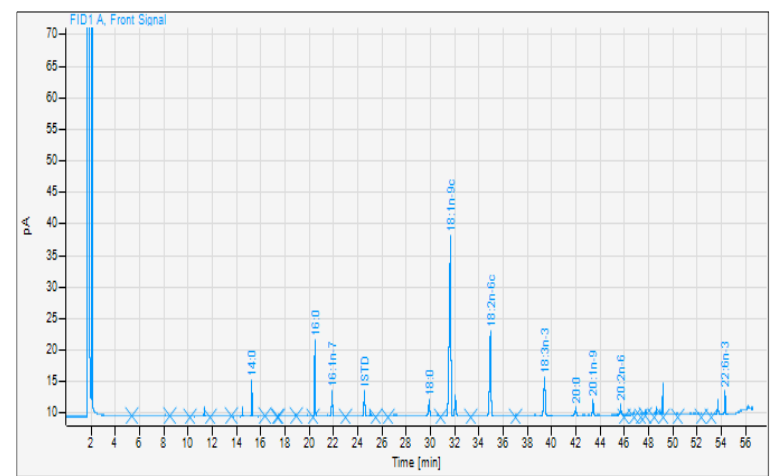

Fig. 9. GC analysis of salmon oil 


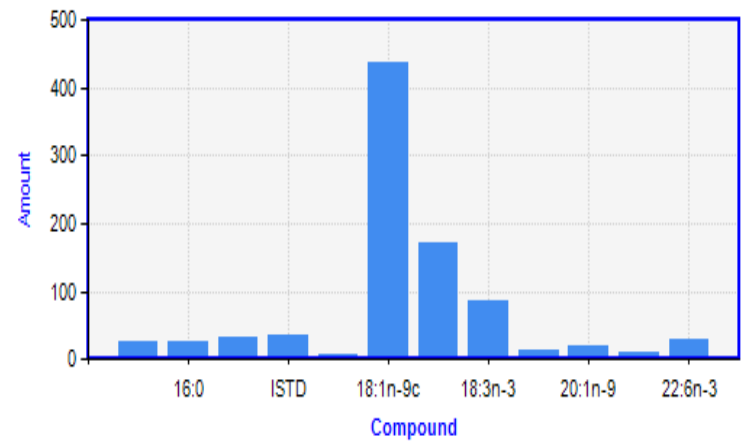

Fig. 10. Amount of different fatty acids: Salmon oil

\subsubsection{Different classifications of fatty acids in trout oil}

The trout oil is composed of a 15 different fatty acids, principally the oleic acid (49.2\%), linoleic acid (18.44\%) and docosahexaénoïque acid $(6.78 \%)$. It is also composed of erucic acid but with a small amount(2.9\%) see figure 11 and 12 .

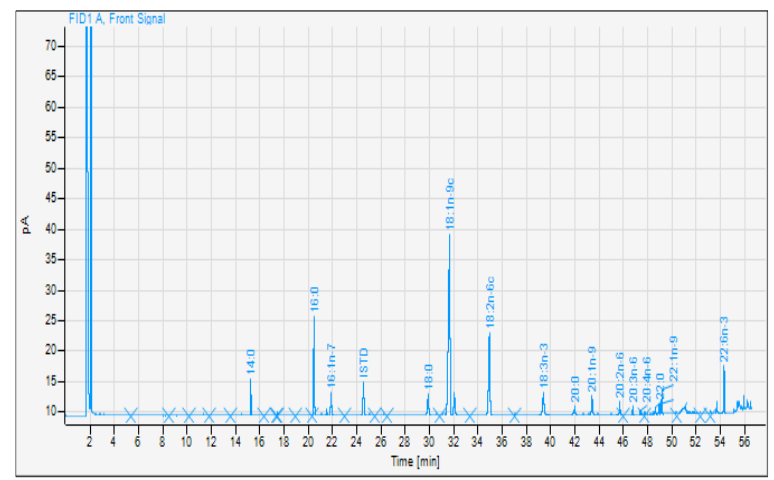

Fig. 11.GC analysis of trout oil

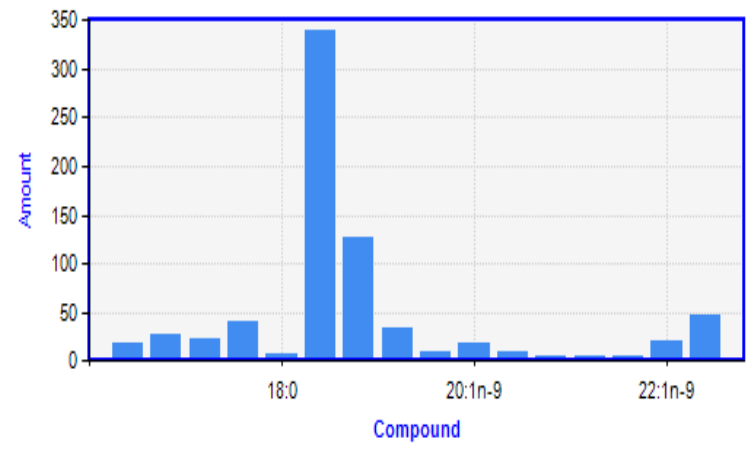

Fig. 12. Amount of different fatty acids: Trout oil

\subsubsection{Different classifications of fatty acids in sardine oil}


The erucic acid(18.56\%), oleic acid (15.87\%), docosahexaénoïque acid (13.58\%) and Butyric acid $(12.52 \%)$ are the major fatty acids from 14 different classes of fatty acids in sardine oil.

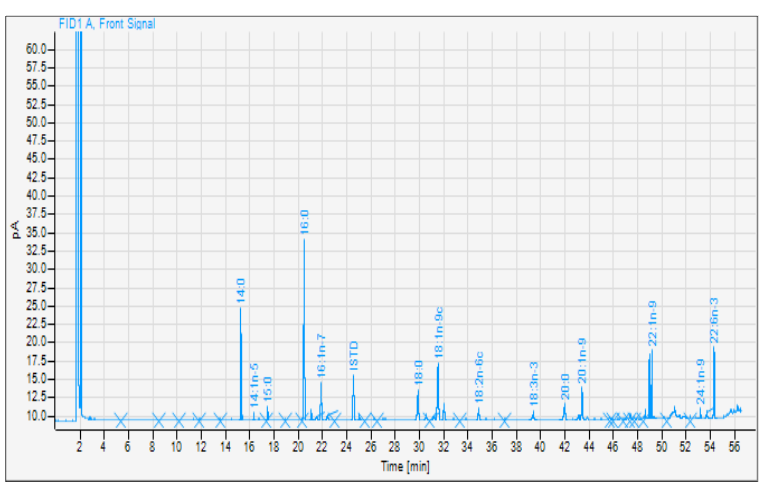

Fig. 13. GC analysis of Sardine oil

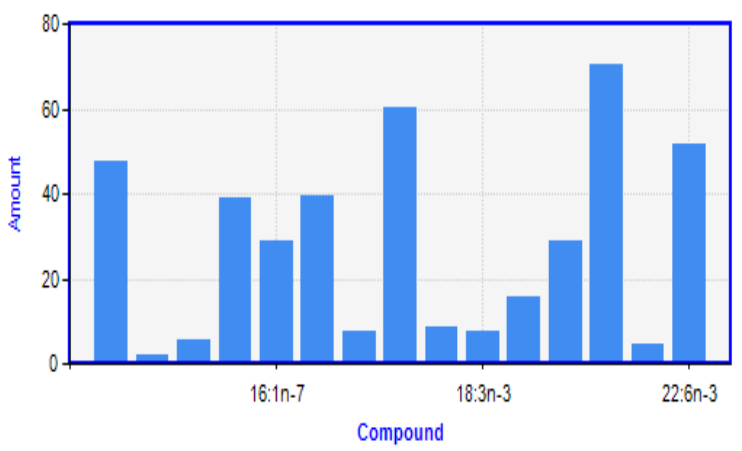

Fig. 14. Amount of different fatty acids: Sardine oil

\subsubsection{Total fatty acids in salmon, trout and sardine oil}

The salmon by-products contain the highest percentage of total fatty acids (figure15). For this reason, the following steps ( transesterifcation and chemical and physicochemical analyses) will be carried out on this type of by-products.

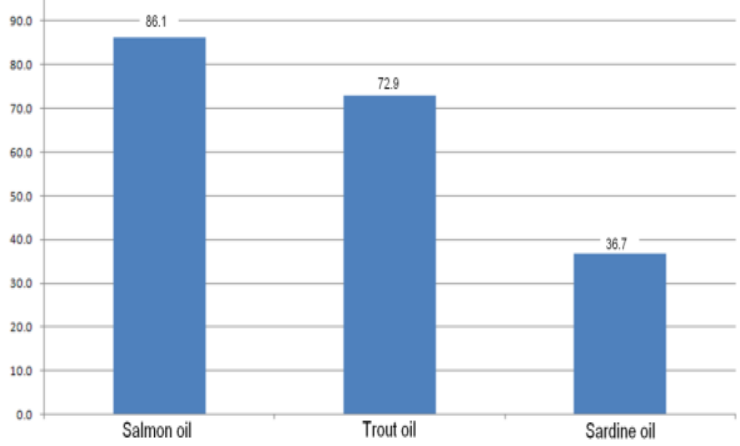

Fig. 15. Percentage of total fatty acids for the 3 fish oil types 


\subsection{The transesterification reaction}

In previous studies, The basic transesterification yield of the high erucic acid content oil is low because of the soap formation so it provides a minimum yield of ester. In addition, the biodiesel from the low erucic acid content oil has the same properties, compared to diesel specifications[6]. On the basis of this study, we chose the salmon oil that has the lowest erucic acid content for the transesterification reaction and biodiesel production.

After the transesterification of salmon oil and after mixture settling, two separate phases are obtained. A lower phase containing glycerin which is immiscible with esters settles at the bottom of the funnel. A higher phase that contains esters or biodiesel mixed with excess alcohol see figure 16 .

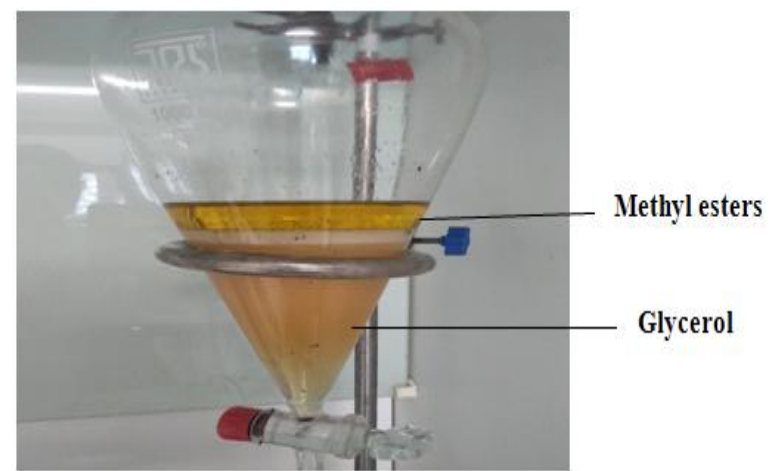

Fig. 16. phase separation after transesterification

A sample of salmon oil and its methyl esters after transesterification were analysed by highperformance thin-layer chromatography (HPTLC). The results of this analysis show the disappearance of triglycerides after transesterification ( Figure17 and 18), which validates the transesterification reaction.

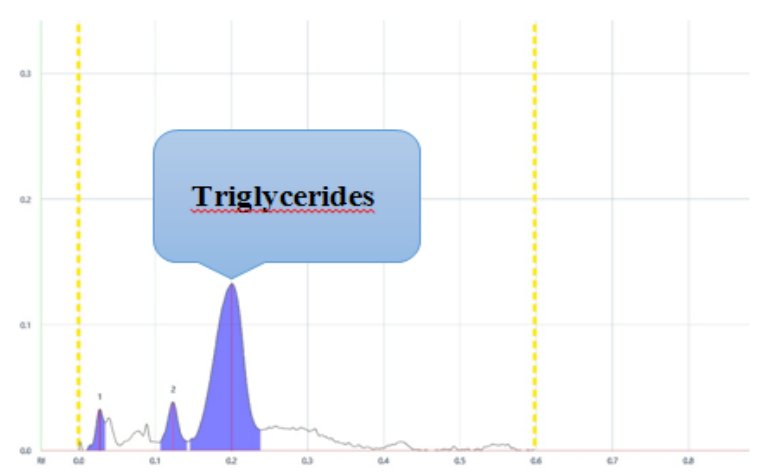

Fig. 17. HPTLC of salmon oil 


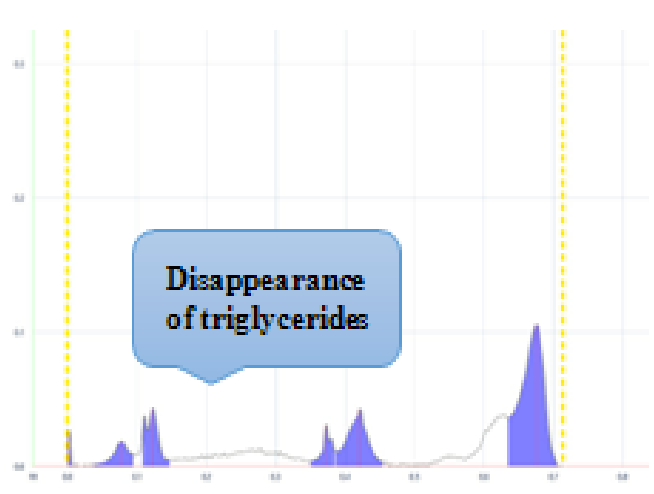

Fig. 18. HPTLC of methyl esters

\section{Conclusion and perspectives}

The reactive extrusion process is used in order to extract the oil from the fish coproducts. Experimental tests confirm that the used of this process is more efficient compared to the batch process, for many reasons. It offers an important raw material denaturation and a better oil recovery while minimizing the pellet phase. In addition, the used of this process for the fish coproducts hydrolysis compared to batch process optimise the process parameters; the extraction time is reduce from $60 \mathrm{~min}$ by using the batch to only $5 \mathrm{~min}$.

It is therefore more advantageous to use salmon byproducts as a raw material for biodiesel production. The salmon recovered oil percentage is more important than the other recovered oil types ( trout and sardine). The salmon oil is chosen for its characteristics, it contains the highest percentage of lipids. Futhermore, It has the lowest erucic acid content allowing a viable transesterification so a good ester yield. Besides, the extracted biodiesel from the lowest erucic content oil has a similar properties as the diesel.

The further tests will focus on the determination of the acidity index, chemical composition, viscosity, density of methyl esters. The same analyses will be carried out for diesel in order to compare the parameters. The effect of the different biodiesel and diesel mixture on the engine performances will be subject of future studies.

\section{References}

[1] Drvodelic, N.: Analyse de biodiesel selon les normes ASTM D6751 et EN 14214 à 1 ' aide de 1' ICP-OES Agilent 5100 SVDV. (2014)

[2] Adewale, P., Dumont, M., and Ngadi, M.: Recent trends of biodiesel production from animal fat wastes and associated production techniques. Renew. Sustain. Energy Rev. vol. 45, pp. 574-588 (2015)

[3] Sajjadi, B., Aziz, A., Raman, A. and Arandiyan, H.: A comprehensive review on properties of edible and non-edible vegetable oil-based biodiesel: Composition, speci fi cations and prediction models. Renew. Sustain. Energy Rev. vol. 63, pp. 62-92 (2016)

[4] Amini, Z., Chyuan, H., Harrison, M. D., Kusumo, F., Mazaheri, H. and Ilham, Z.: Biodiesel production by lipase-catalyzed transesterification of Ocimum basilicum L . ( sweet basil ) seed oil. Energy Convers. Manag. vol. 132, pp. 82-90 (2017)

[5] Nisar, J., Razaq, R., Farooq, M., Iqbal, M., Ali, R., Sayed, M. and Shah, A.: Enhanced biodiesel 
production from Jatropha oil using calcined waste animal bones as catalyst. Renew. Energy. vol. 101, pp. 111-119 (2017)

[6] Dorado, M. P., Ballesteros, E., Lo, F. J. and Mittelbach, M.: Optimization of Alkali-Catalyzed Transesterification of Brassica Carinata Oil for Biodiesel Production. Energy \& Fuels. vol. 18, pp. 77$83(2004)$ 